\title{
Nuclear Factor (Erythroid-Derived)-Related Factor 2-Associated Retinal Pigment Epithelial Cell Protection under Blue Light-Induced Oxidative Stress
}

\author{
Kei Takayama, ${ }^{1}$ Hiroki Kaneko, ${ }^{1}$ Keiko Kataoka, ${ }^{1}$ Reona Kimoto, ${ }^{1}$ \\ Shiang-Jyi Hwang, ${ }^{1,2}$ Fuxiang Ye, ${ }^{1,3}$ Yosuke Nagasaka, ${ }^{1}$ Taichi Tsunekawa, ${ }^{1}$ \\ Toshiyuki Matsuura, ${ }^{1}$ Norie Nonobe, ${ }^{1}$ Yasuki Ito, ${ }^{1}$ and Hiroko Terasaki ${ }^{1}$ \\ ${ }^{1}$ Department of Ophthalmology, Nagoya University Graduate School of Medicine, Nagoya 466-8550, Japan \\ ${ }^{2}$ Laboratory of Bell Research Center-Department of Obstetrics and Gynecology Collaborative Research, \\ Nagoya University Graduate School of Medicine, Nagoya 466-8550, Japan \\ ${ }^{3}$ Department of Ophthalmology, Shanghai First People's Hospital, Shanghai Jiao Tong University School of Medicine, \\ Shanghai 200240, China
}

Correspondence should be addressed to Hiroki Kaneko; h-kaneko@med.nagoya-u.ac.jp

Received 28 May 2016; Revised 29 July 2016; Accepted 25 August 2016

Academic Editor: Ian Copple

Copyright (C) 2016 Kei Takayama et al. This is an open access article distributed under the Creative Commons Attribution License, which permits unrestricted use, distribution, and reproduction in any medium, provided the original work is properly cited.

\begin{abstract}
Purpose. It is a matter of increasing concern that exposure to light-emitting diodes (LED), particularly blue light (BL), damages retinal cells. This study aimed to investigate the retinal pigment epithelium (RPE) damage caused by BL and to elucidate the role of nuclear factor (erythroid-derived)-related factor 2 (Nrf2) in the pathogenesis of BL-induced RPE damage. Methods. ARPE-19, a human RPE cell line, and mouse primary RPE cells from wild-type and Nrf2 knockout ( $\mathrm{Nrf2^{-/- }}$ ) mice were cultured under blue LED exposure (intermediate wavelength, $450 \mathrm{~nm}$ ). Cell death rate and reactive oxygen species (ROS) generation were measured. TUNEL staining was performed to detect apoptosis. Real-time polymerase chain reaction was performed on NRF2 mRNA, and western blotting was performed to detect Nrf2 proteins in the nucleus or cytoplasm of RPE cells. Results. BL exposure increased cell death rate and ROS generation in ARPE-19 cells in a time-dependent manner; cell death was caused by apoptosis. Moreover, BL exposure induced NRF2 mRNA upregulation and Nrf2 nuclear translocation in RPE. Cell death rate was significantly higher in RPE cells from $\mathrm{Nrf2}^{-/-}$mice than from wild-type mice. Conclusions. The Nrf2 pathway plays an important role in protecting RPE cells against BL-induced oxidative stress.
\end{abstract}

\section{Introduction}

Age-related macular degeneration (AMD) leads to blindness, accounting for approximately $9 \%$ of blindness cases worldwide. There are approximately 30 million patients with AMD, of whom $>0.5$ million have become blind [1]. AMD can be divided into two categories: wet and dry AMD [2]. In wet AMD, choroidal neovascularization breaks the retinal pigment epithelium (RPE) through to the neural retina, causing the leakage of fluid, lipids, and blood; these changes lead to fibrous scarring and antivascular endothelial growth factor drugs are approved for wet AMD treatment [3-5]. In dry AMD, progressive geographic atrophy of RPE occurs, followed by severe damage of the photoreceptors. Severe irreversible blindness from AMD is caused by these advanced forms $[6,7]$.

AMD may have a multifactorial pathogenesis [8] and is characterized by photoreceptor cell death [9-14]. Several factors, such as smoking, obesity, eating habits, and light exposure, particularly blue light (BL) exposure, play important roles in the progression of AMD [15-18]. BL exposure causes an increase in the reactive oxygen species (ROS), which may result in structural damage and decreased viability of retinal cells. It also causes RPE apoptosis via oxidative stress and mitochondrial damage [19-21]. 
One of the most important antioxidation pathways is the nuclear factor (erythroid-derived)-like 2 (Nrf2) pathway. $\mathrm{Nrf2}$ is a $65 \mathrm{kDa}$ molecule with a basic leucine zipper structure [22]. Without oxidative stress, Nrf2 in its inactive state is bound to Kelch-like ECH-associated protein 1 (Keap1) in the cytoplasm [23]. When cells are exposed to oxidative stress, the active site cysteine residues of Keapl are oxidized, preventing Keap1 from interacting with NRF2. With Nrf2 accumulation in the cytoplasm, Nrf2 moves to the nucleus and binds to the antioxidant response element [24]. Nrf2 also serves as the master regulator of a highly coordinated antioxidant response in RPE cells [25]. Several studies demonstrated that antioxidative factors prevent RPE cells from being damaged by oxidative stress [26-34]. Some antioxidative factors also upregulate Nrf2 signaling. RPE damage may be prevented by these antioxidative factors via the upregulation of Nrf2 signaling. However, little is known regarding whether Nrf2 signaling activation is directly involved in RPE protection. Moreover, to the best of our knowledge, the direct relationship between BL exposure and Nrf2 signaling in RPE cells has not been well elucidated.

$\mathrm{Nrf2}$ knockout $\left(\mathrm{Nrf2} 2^{-/-}\right)$mice have been used in studies on systemic and ocular diseases. In ophthalmology, there are some studies regarding simulating diabetic retinopathy, ischemic retinopathy, and macular degeneration [32, 35-40]. $\mathrm{Nrf2}^{-/-}$mice developed ocular pathology similar to AMD [35]. Moreover, primary RPE cells from $\mathrm{Nrf2} 2^{-/-}$mice are susceptible to oxidative stress [32]. However, to the best of our knowledge, no studies have focused on $\mathrm{Nrf2^{-/- }}$ RPE cells exposed to BL. Therefore, in this study, we prepared primary RPE cells from $N r f 2^{-/-}$mice and investigated the direct involvement of Nrf2 signaling in BL-induced RPE cell damage.

\section{Materials and Methods}

2.1. Cell Culture, Primary Cell Preparation, and BL Exposure. ARPE-19, a human RPE cell line, was purchased from the American Type Culture Collection (Rockville, MD, USA), and primary human RPE (hRPE) cell line was purchased from Lonza (Walkersville, MD, USA). Cells were grown in colorless Dulbecco's modified Eagle's medium (DMEM) premixed with Ham's F-12 (1:1 ratio, Sigma-Aldrich) and supplemented with $10 \%$ fetal bovine serum and the antibiotics streptomycin/penicillin G (Sigma-Aldrich) [13, 14]. Primary mouse RPE cells were collected from the wild-type and $\mathrm{Nrf2} 2^{-/-}$mice, as previously described [41, 42]. In brief, mouse eyecups were washed with sterile PBS, and flatmounts were created. The retina was gently removed to allow RPE layer to be on the surface of the flatmount. The RPE eyecups were rinsed in a chelating agent (Versene, Invitrogen), and RPE cells were enzymatically dislodged by $2 \%$ Dispase (Roche Diagnostics). Dislodged RPE cells were collected and cultured in DMEM containing antibiotics at $37^{\circ} \mathrm{C}$ containing $5 \% \mathrm{CO}_{2}$. Animal studies were approved by the Institutional Animal Care and Use Committee of the Nagoya University Graduate School of Medicine. All procedures involving animals were conducted according to the Association for
Research in Vision and Ophthalmology Statement for the Use of Animals in Ophthalmic and Vision Research. Nrf2 $2^{-/-}$mice were provided by RIKEN BRC through the National BioResource Project of MEXT, Japan [43]. The cells were cultured in the dark or under BL (Zensui LED Lamp Blue ${ }^{\mathrm{Tm}}$; Zensui Inc., Japan; peak wavelength: $450 \mu \mathrm{m}, 1,200$ lux).

2.2. LDH Assay and ROS Measurement. The cell death rate was evaluated by measuring the lactate dehydrogenase (LDH) activities using the Cytotoxicity Detection Kit PLUS (Roche Diagnostics, Mannheim, Germany). The supernatant of the culture medium, which contained LDH secreted from dead cells, was collected, followed by the addition of Triton X100 in the medium to release intracellular $\mathrm{LDH}$ from the surviving cells. After measuring the LDH activities in the culture supernatant and medium, the proportions of dead cells among the total cells were calculated. Oxidative stress on BL exposure was evaluated with respect to the amount of ROS, measured using the OxiSelect ${ }^{\mathrm{TM}}$ ROS assay kit (Cell Biolabs. Co., Japan). In brief, after BL exposure, the assay was terminated by adding cell lysis buffer, and fluorescence intensity was measured at $493 \mathrm{~nm}(\mathrm{ex}) / 523 \mathrm{~nm}$ (em) using a fluorescent plate reader at each time point.

2.3. Cell Morphology and TUNEL Staining. Morphological changes of ARPE-19 cells exposed to BL were visualized using a phase-contrast microscope (FSX-100; Olympus, Tokyo, Japan). TUNEL-positive apoptotic cells were detected, as previously described [14, 44]. In brief, after $24 \mathrm{~h}$ of $\mathrm{BL}$ exposure, the cells were fixed with $2 \%$ PFA for $20 \mathrm{~min}$ at room temperature on the chambered cell culture slides. The cells were stained with the In Situ Cell Death Detection kit (Roche Diagnostics, Mannheim, Germany) and $0.3 \mathrm{mg} / \mathrm{mL} 4^{\prime}, 6$ diamidino-2-phenylindole (Invitrogen, Carlsbad, CA, USA) for $1 \mathrm{~h}$. The stained cells were then observed using a Bio Imaging Navigator fluorescence microscope (BZ-9000; Keyence, Osaka, Japan). The number of TUNEL-positive cells was calculated from images obtained with a $20 \mathrm{x}$ lens $(537 \times 710 \mu \mathrm{m})$. The average number of TUNEL-positive cells observed in three independent areas was calculated per well $(n=$ number of wells) [14].

2.4. Protein and RNA Isolation. For total protein collection, the cultured human and mouse cells were lysed in RIPA buffer (Sigma-Aldrich) with a protease inhibitor cocktail (Roche Diagnostics, Indianapolis, IN, USA). The lysate was centrifuged at $15,000 \times \mathrm{g}$ for $15 \mathrm{~min}$ at $4^{\circ} \mathrm{C}$, and the supernatant was collected. Protein concentrations were determined using the Bradford Assay Kit (Bio-Rad, Hercules, CA, USA) with bovine serum albumin as the standard. To measure Nrf2 abundance in the nucleus, ARPE-19 cells were treated with NE-PER Nuclear and Cytoplasmic Extraction Kit (Pierce, Rockford, IL, USA) as previously described [41]. For realtime polymerase chain reaction (RT-PCR) analyses, total RNA was purified using the Qiagen RNeasy Mini Kit (Qiagen, Hilden, Germany), according to the manufacturer's protocol; the RNA concentration and quality were assessed using the 
NanoDrop ND-1000 spectrophotometer (NanoDrop Technologies, Rockland, DE, USA) [13].

2.5. Quantitative Reverse Transcription-PCR (RT-PCR). The total RNA was reverse transcribed using the Transcriptor Universal cDNA Master Kit (Roche Diagnostics), starting with $2 \mu \mathrm{g}$ of total RNA from each sample [13]. RT-PCR was performed using the Thunderbird Probe qPCR Mix (Toyobo Life Science, Osaka, Japan) and Gene Expression Assay containing primers and an FAM dye-labeled TaqMan probe for detecting human NRF2 (HS00965961-gl; Applied Biosystems, USA) and eukaryotic 18S rRNA (Hs_99999901_s1; Applied Biosystems) that is available for human 18S rRNA [12]. PCR cycles consisted of a predenaturation step at $95^{\circ} \mathrm{C}$ for $2 \mathrm{~min}$ followed by 40 cycles of denaturing steps at $95^{\circ} \mathrm{C}$ for $15 \mathrm{~s}$ and annealing and extending steps at $60^{\circ} \mathrm{C}$ for $60 \mathrm{~s}$. The relative expressions of the target genes were determined using the $2^{-\Delta \Delta \mathrm{Ct}}$ method.

2.6. Western Blotting. Western blotting was performed as previously described $[13,14]$. In brief, proteins $(50 \mu \mathrm{g})$ from ARPE-19 cells were run on SDS precast gels (Wako, Osaka, Japan) and were transferred to PVDF membranes. The transferred membranes were washed with TBS-T $(0.05 \mathrm{M}$ Tris, $0.138 \mathrm{M} \mathrm{NaCl}, 0.0027 \mathrm{M} \mathrm{KCl}, \mathrm{pH}=8.0$, and $0.05 \%$ Tween 20; Sigma-Aldrich) and then blocked with $5 \%$ nonfat dry milk/TBS-T at room temperature for $2 \mathrm{~h}$. The membranes were then incubated with the rabbit antibody against NRF2 $\left(1: 100\right.$; Santa Cruz Biotechnology) at $4^{\circ} \mathrm{C}$ overnight. Total protein loading was assessed by immunoblotting using $\beta$ actin (1:3000: Cell Signaling), and nuclear protein loading was assessed by immunoblotting using lamin B (rabbit, 1:200; Santa Cruz Biotechnology) [45]. HRP-linked secondary antibody was used $(1: 3,000$, Invitrogen) at RT for $1 \mathrm{~h}$. The signal was visualized with enhanced chemiluminescence (ECL plus; GE Healthcare, Piscataway, NJ, USA) and captured using ImageQuant LAS-4000 Imager (GE Healthcare).

2.7. Outcomes and Statistical Analysis. Cell death rate, ROS generation, and RT-PCR of Nrf2 mRNA were statistically analyzed using the Mann-Whitney $U$ test. $P$ values of $<0.05$ were considered to be statistically significant.

\section{Results}

First, we examined whether BL exposure induced ROS generation and RPE cell death. BL exposure caused ARPE19 cells to release $\mathrm{LDH}$. The values of BL-induced/total LDH were $12.0 \% \pm 4.2 \%(n=4), 14.4 \% \pm 5.9 \%(n=4), 25.3 \% \pm$ $5.8 \%(n=4)$, and $27.7 \% \pm 5.7 \%(n=4)$ at $1,2,4$, and $6 \mathrm{~h}$ after BL exposure, respectively. Among these values, there were significant increases at $4 \mathrm{~h}(P=0.012)$ and $6 \mathrm{~h}(P=$ 0.0012 ) compared with the value at $1 \mathrm{~h}$ after BL exposure. In contrast, the values of BL-free/total $\mathrm{LDH}$ were $10.6 \% \pm 3.8 \%$ $(n=4), 10.6 \% \pm 2.3 \%(n=4), 11.6 \% \pm 2.0 \%(n=4)$, and $11.8 \% \pm 4.8 \%(n=4)$ at $1,2,4$, and $6 \mathrm{~h}$ after the onset of treatment, respectively. Among these values, there were also significant differences at $4 \mathrm{~h}(P=0.014)$ and $6 \mathrm{~h}(P=0.0057)$ compared with those without $\mathrm{BL}$ exposure. These findings also showed that ARPE-19 cell death was promoted by BL exposure in a time-dependent manner. This was confirmed by the $\mathrm{LDH}$ results from ARPE-19 cells not exposed to BL (Figure 1(a)). BL exposure also induced ROS generation in ARPE-19 cells in a time-dependent manner. ROS generation was significantly higher at $4 \mathrm{~h}(1.10 \pm 0.05, n=5, P=0.027)$, $6 \mathrm{~h}(1.32 \pm 0.11, n=5, P=0.043)$, and $24 \mathrm{~h}(22.4 \pm 3.2$, $n=5, P<0.001)$ than at $1 \mathrm{~h}(1.0 \pm 0.06, n=5)$ after BL exposure (Figure 1(b)). Compared with ARPE-19 cells at $24 \mathrm{~h}$ after the onset of the treatment without $\mathrm{BL}$ exposure (control, $1.00 \pm 0.10, n=8$ ), ARPE-19 cells exposed to BL generated significantly higher amounts of ROS (Figure 1(c); $14.8 \pm 2.1, n=8, P<0.001)$. In hRPE cells, the values of BLinduced/total LDH were 9.5\% $\pm 2.9 \%(n=4), 8.6 \% \pm 5.9 \%$ $(n=4), 16.4 \% \pm 6.7 \%(n=4)$, and $22.3 \% \pm 5.7 \%(n=4)$ at $1,2,4$, and $6 \mathrm{~h}$ after BL exposure, respectively. Among these values, there were significant increases at $4 \mathrm{~h}(P=0.034)$ and $6 \mathrm{~h}(P=0.0011)$ compared with the value at $1 \mathrm{~h}$ after $\mathrm{BL}$ exposure. In contrast, the values of BL-free/total LDH were $9.4 \% \pm 4.4 \%(n=4), 9.5 \% \pm 4.1 \%(n=4), 11.2 \% \pm 1.6 \%(n=$ $4)$, and $9.4 \% \pm 4.4 \%(n=4)$ at $1,2,4$, and $6 \mathrm{~h}$ after the onset of treatment, respectively. Among these values, there were significant differences at $4 \mathrm{~h}(P=0.048)$ and $6 \mathrm{~h}(P<0.001)$ compared with those without $\mathrm{BL}$ exposure. These findings also showed that hRPE cell death was promoted by BL exposure in a time-dependent manner. This was confirmed by LDH results from hRPE cells not exposed to BL (Figure 1(d)). BL exposure also induced ROS generation in hRPE cells. ROS generation was significantly higher at $4 \mathrm{~h}(3.23 \pm 0.07, n=8$, $P<0.001), 6 \mathrm{~h}(33.28 \pm 0.49, n=8, P<0.001)$, and $24 \mathrm{~h}$ $(10.54 \pm 0.41, n=8, P<0.001)$ than at $1 \mathrm{~h}(1.0 \pm 0.06$, $n=8$ ) after BL exposure (Figure 1(e)). Compared with hRPE cells at $24 \mathrm{~h}$ after the onset of the treatment without BL exposure (control, $1.00 \pm 0.07, n=8$ ), hRPE cells exposed to BL generated significantly higher amounts of ROS (Figure 1(f); $22.12 \pm 0.91, n=8, P<0.001)$.

Moreover, we examined the morphological change of ARPE-19 cells on BL exposure. Under normal condition, ARPE-19 cells showed a spindle shape. However, on BL exposure, these cells shrank and changed to an oval shape. These findings indicated that $\mathrm{BL}$ exposure decreased the viability of ARPE-19 cells as previously described (Figure 2) [46]. To clarify more precisely the mechanism of ARPE-19 cell death by BL exposure, we performed TUNEL staining of ARPE-19 cells with and without BL exposure. This staining revealed that numerous ARPE-19 cells were TUNEL-positive at $24 \mathrm{~h}$ after $\mathrm{BL}$ exposure. These findings indicated that BL exposure-induced ARPE-19 cell death mostly involved apoptosis (Figure 3).

To investigate the involvement of NRF2 in the effect of BL exposure on ARPE-19 cells, we examined mRNA level and NRF2 protein expression of ARPE-19 cells with and without BL exposure. Compared with the mRNA level of ARPE-19 cells at the onset of treatment (control, $1.00 \pm 0.09$, $n=2$ ), the level at $6 \mathrm{~h}$ after BL exposure was significantly higher (Figure 4(a); $7.24 \pm 3.74, n=8, P=0.042$ ). NRF2 mRNA level was increased by BL exposure, whereas it did not significantly increase in the absence of BL exposure from 0 to $6 \mathrm{~h}$ after the onset of treatment. Nrf2 protein 


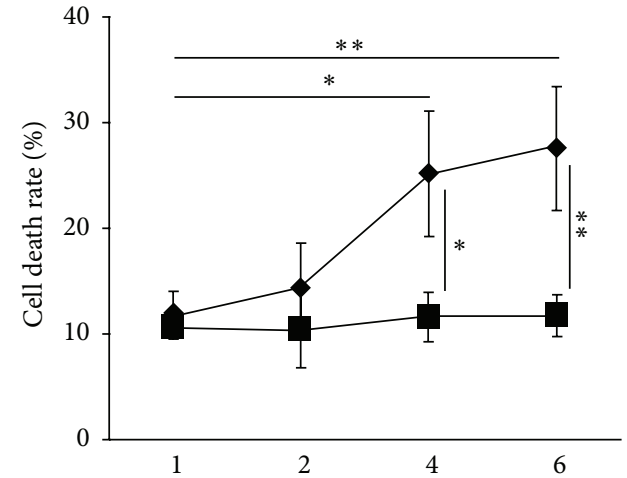

(h)

$$
\multimap \mathrm{BL}(+)
$$$$
\text { BL(-) }
$$

(a)

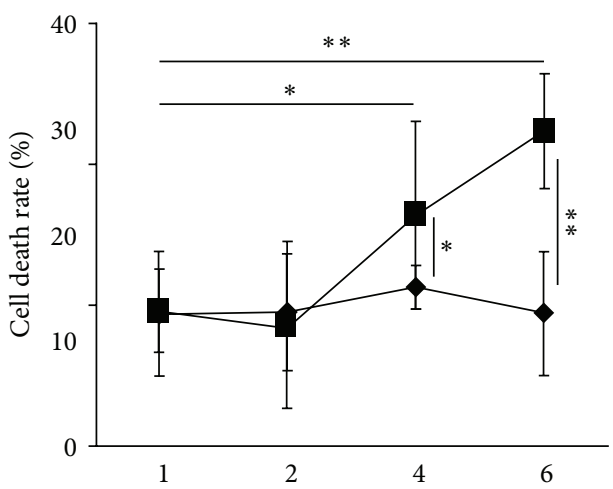

(h)

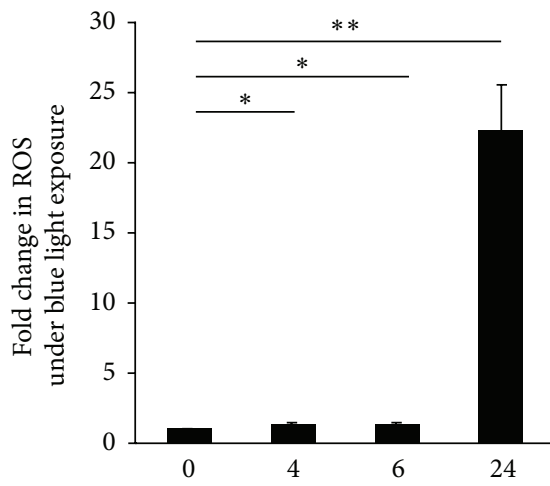

(h)

(b)

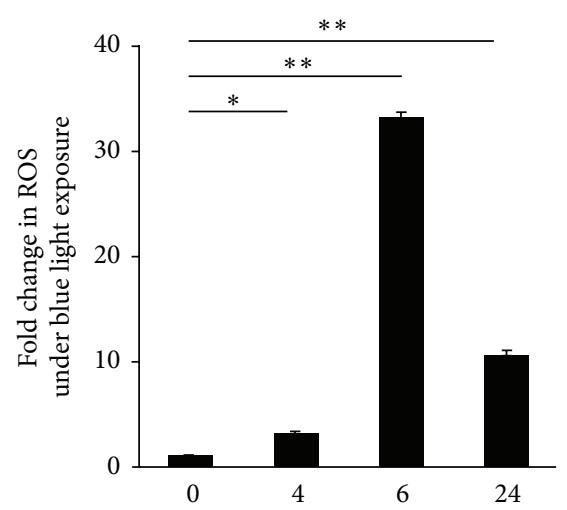

(h)

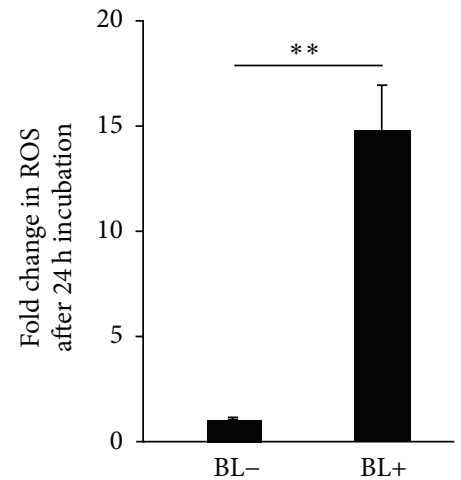

$\mathrm{BL}-$

(c)

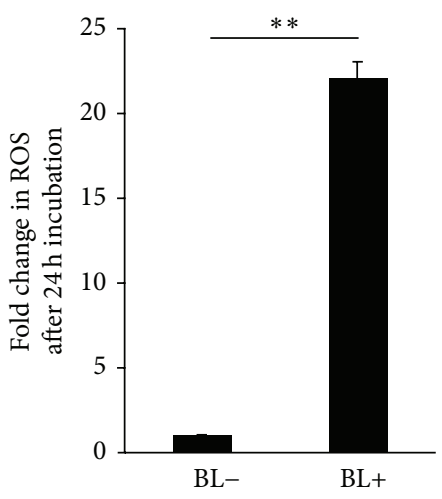

(e)

(f)

FIGURE 1: Increases in cell death rate and ROS generation in ARPE-19 cells and human RPE (hRPE) cells with or without BL exposure. (a) BL exposure increased the cell death rate and (b) ROS generation in ARPE-19 cells exposed to BL in a time-dependent manner. (c) ROS reactivity in ARPE-19 cells was significantly higher with BL exposure than without BL exposure $24 \mathrm{~h}$ later. (d) BL exposure increased the cell death rate and (e) ROS generation in hRPE cells exposed to BL. (f) ROS reactivity in hRPE cells was significantly higher with BL exposure than without BL exposure 24 h later. ${ }^{*} P<0.05 ;{ }^{* *} P<0.01$.

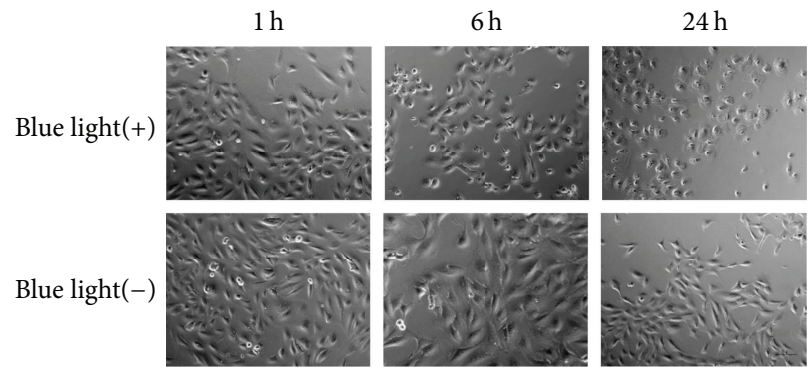

FIGURE 2: Morphological changes of ARPE-19 cells with or without blue light (BL) exposure. ARPE-19 cells changed to an oval shape and shrank upon BL exposure in a time-dependent manner $(1,6$, and $24 \mathrm{~h})$, whereas only minor changes were shown in those without BL exposure. Scale bar $=100 \mu \mathrm{m}$. 

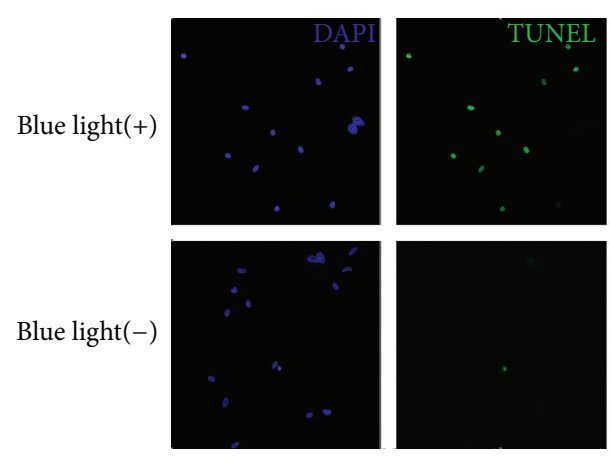

(a)

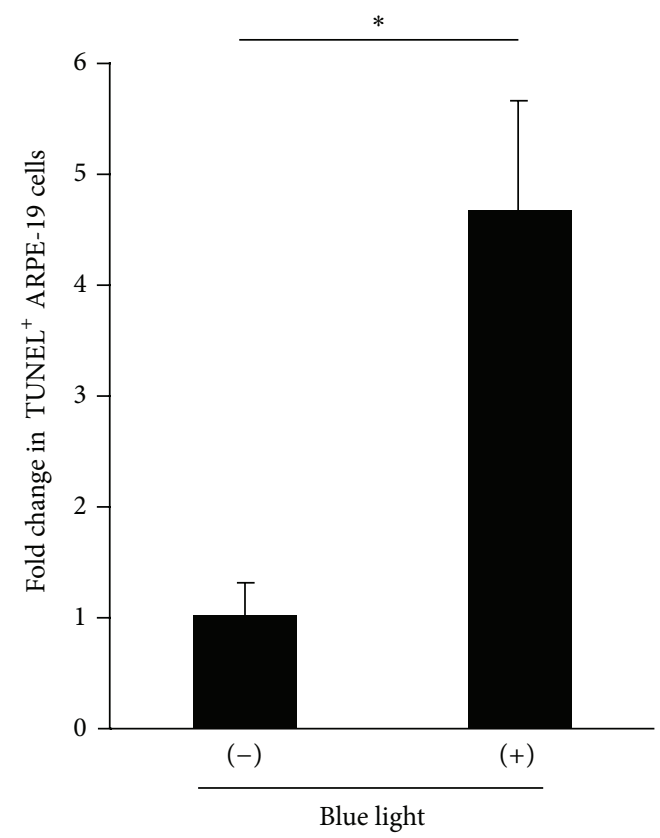

(b)

FIGURE 3: Detection of apoptosis in ARPE-19 cells at $24 \mathrm{~h}$ with or without BL exposure. (a) TUNEL (green) staining showed that, $24 \mathrm{~h}$ after BL exposure, ARPE-19 cells showed TUNEL positivity, reflecting apoptosis, by BL exposure. (b) There was a significant increase (4.67-fold) in the number of TUNEL-positive RPE cells with BL exposure compared to those without BL exposure. DAPI indicates the nucleus. Scale bar $=100 \mu \mathrm{m} .{ }^{*} P<0.01$.

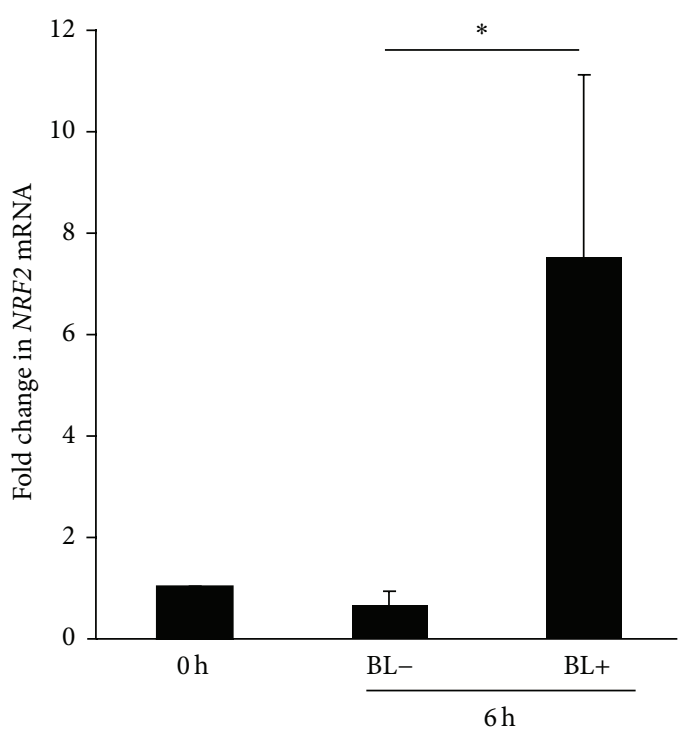

(a)

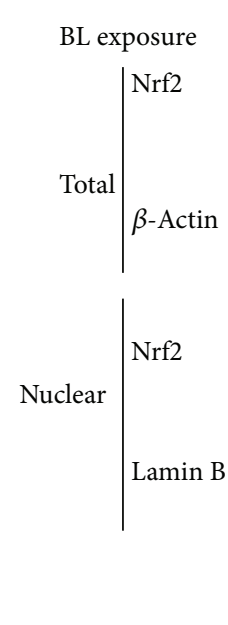

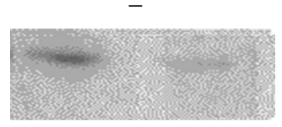
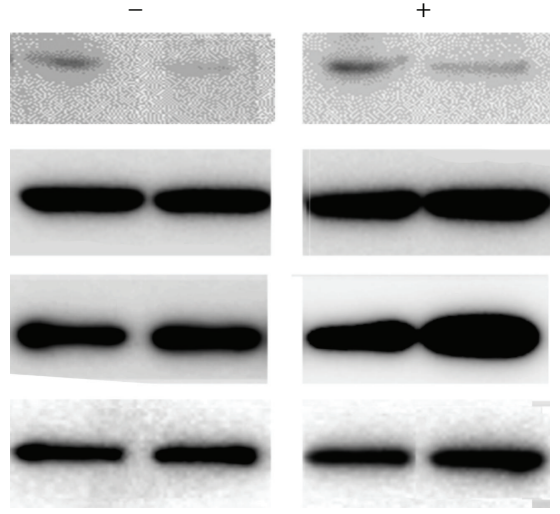

$6 \mathrm{~h}$
$6 \mathrm{~h}$

(b)

FIGURE 4: Nrf2 abundance in ARPE-19 cells with blue light (BL) exposure. (a) BL exposure increased NRF2 mRNA level in ARPE-19 cells and showed a significant difference at $6 \mathrm{~h}$ compared with that without BL exposure. (b) Nrf2 protein in the total ARPE-19 cells was decreased at $6 \mathrm{~h}$ of exposure, although Nrf2 protein in the nucleus was increased at $6 \mathrm{~h}$ of exposure. ${ }^{*} \mathrm{P}<0.05$.

expression from total ARPE-19 cells appeared to be decreased by BL exposure (Figure 4(b)). In an active state, Nrf2 showed nuclear translocation. Therefore, we separately obtained Nrf2 protein from the nucleus of ARPE-19 cells. Nrf2 protein was abundantly expressed in the ARPE-19 nucleus (Figure 4(b)).
These results showed that, as a result of BL exposure, Nrf2 in ARPE-19 cells was activated. To shed more light on the importance of Nrf2 activation in BL exposure-induced ARPE-19 cell damage, we collected primary RPE cells from $\mathrm{Nrf2} 2^{-/-}$and wild-type $\left(\mathrm{Nrf2} 2^{+/+}\right)$mice and compared their cell 


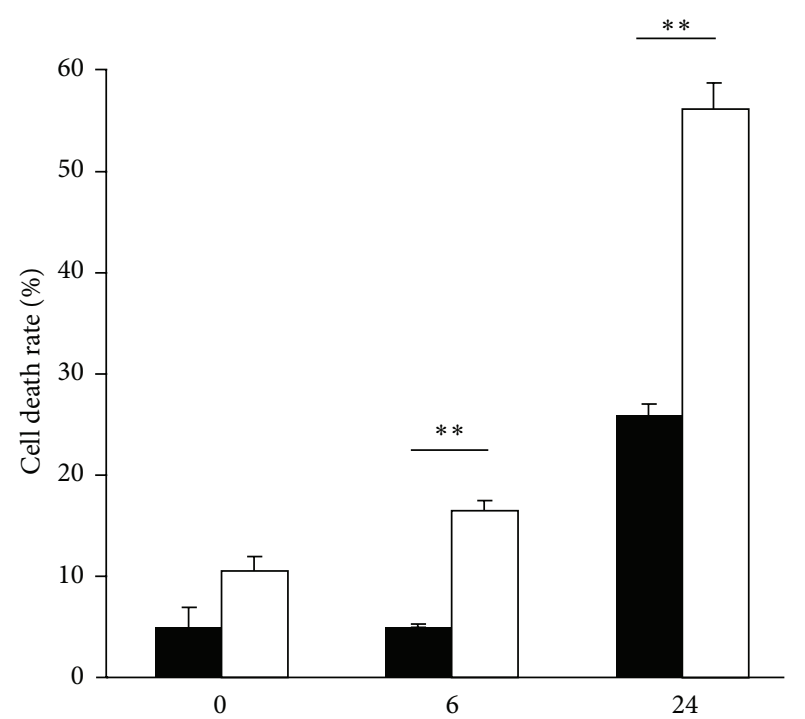

(h)

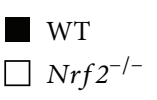

FIGURE 5: Increased cell death in $\mathrm{Nrf}^{-/-} \mathrm{RPE}$ cells under blue light (BL) exposure. BL exposure induced more cell death in $\mathrm{Nrf2}^{-/-} \mathrm{RPE}$ cells than in wild-type RPE cells. ${ }^{* *} P<0.01$.

death rates on $\mathrm{BL}$ exposure. Under these conditions, death rates of RPE cells from $N r f 2^{-/-}$mice were $16.4 \pm 1.1(n=6)$ and $56.1 \pm 2.8(n=6)$ at 6 and $24 \mathrm{~h}$ after exposure, respectively. In contrast, death rates of RPE cells from wild-type mice were $5.0 \pm 0.1(n=6)$ and $25.7 \pm 1.4(n=6)$ at 6 and $24 \mathrm{~h}$ after exposure, respectively. There were significant increases in the death rate in $\mathrm{Nrf2^{-/- }}$ mouse RPE cells at $6 \mathrm{~h}(P<0.001)$ and $24 \mathrm{~h}(P<0.001)$ compared with those in wild-type mice (Figure 5). These findings indicated that Nrf2 plays an important role in blocking cell death caused by BL exposureinduced ROS generation.

\section{Discussion}

Visible light exposure-induced damage in retinal cells occurs through type I (free radical) and type II (oxygen-dependent) mechanisms. Free radicals induce cells to undergo necrosis, whereas oxygen-dependent mechanisms induce them to undergo apoptosis [47]. The mechanism of AMD was considered to involve oxidative stress caused by several factors driving RPE cells to die via apoptosis. BL exposure induces ROS generation, damaging mitochondrial DNA and cell structure; then, RPE cells are forced to enter an apoptotic state [15-17]. In this study, we demonstrated that BL exposure increased ROS generation in RPE cells and induced cell death in a time-dependent manner. In addition, TUNEL staining suggested that apoptotic RPE cell death was caused by $\mathrm{BL}$ exposure. To an extent, these findings simulate the pathogenesis of AMD.

ARPE-19 cells have some differences from primary hRPE cells in terms of promoter strength [48], proliferation, and cell death [49]. Previous studies have demonstrated that ARPE-19 cells are stronger and tolerable for oxidative stress compared with primary hRPE cells. In the present study, cell death rate and ROS of primary hRPE cells at $6 \mathrm{~h}$ of BL exposure (Figures $1(\mathrm{~d})$ and 1(e)) were higher than those of ARPE-19 cells (Figures 1(a) and 1(b)). It has been previously demonstrated that primary hRPE cells are more sensitive than ARPE-19 cells against BL exposure-derived oxidative stress. On the other hand, ROS generation at $24 \mathrm{~h}$ was lower than that at $6 \mathrm{~h}$ in primary hRPE cells. This is possibly because primary hRPE cells were killed by $24 \mathrm{~h}$ BL exposure, and lower ROS generation was measured from the remaining (smaller number of) cells. Although both cells were damaged by $\mathrm{BL}$ exposure-derived ROS in a time-dependent manner, we considered that the use of these cells for this BL exposure study design is suitable.

The Nrf2 pathway is one of the most important pathways for protecting cells against oxidative stress [22]. Without oxidative stress, Nrf2, in its inactive state, is kept in the cytoplasm [23]. When cells are exposed to oxidative stress, this Nrf2 is released from Keap1 and moves to the nucleus where it functions in antioxidative protective mechanisms [24]. Other cells, such as epidermoid carcinoma cells [50] and retinal ganglion cells [51], also showed increased Nrf2 protein expression on BL exposure in vitro. These reports suggested that BL exposure increased ROS generation, activated Nrf2 signaling, and reduced cell viability in a time-dependent manner. In our study, BL exposure of RPE cells increased ROS generation and apoptotic cell death rate in a timedependent manner. In addition, Nrf2 mRNA level and Nrf2 protein expression in the nucleus were increased in RPE cells in a time-dependent manner. These findings indicated that BL exposure induces the upregulation of ROS and Nrf2, which are involved in antioxidative protective mechanisms as previously reported.

If the Nrf2 pathway does not properly function, antioxidative protection would be weaker and oxidative stress would cause severe cell damage. $\mathrm{Nrf2^{-/- }}$ mice developed ocular pathology similar to the cardinal features of human AMD; deregulated autophagy is a likely mechanistic link between oxidative injury and inflammation [35]. Nrf2 $2^{-/-}$ RPE cells are susceptible to oxidative stress induced by tbutylhydroperoxide [32]. However, these studies did not use light exposure as an oxidative stressor. In dermatology, some studies revealing the relationship between $\mathrm{Nrf2^{-/- }}$ cells and light exposure have been published [52-55]. Ultraviolet lightirradiated $\mathrm{Nrf2} 2^{-/-}$cells exhibited accelerated photoaging, resulting in the necrosis of irradiated cells, inflammatory cell infiltration, TUNEL-positive apoptotic cell formation, and the accumulation of oxidative DNA products, which are caused by oxidative stress. In this study, $N r f 2^{-/-}$RPE cells died at rates of $10.5 \%, 16.4 \%$, and $56.1 \%$ at 0,6 , and $24 \mathrm{~h}$ of $\mathrm{BL}$ exposure, respectively. This rate of cell death at $0 \mathrm{~h}$ tended to be higher than that of wild-type RPE cells, and the rates at 6 and 24 h were significantly higher than those of the wildtype RPE cells. These findings suggest that $\mathrm{Nrf2}^{-/-}$RPE cells are weaker than wild-type RPE cells, and Nrf2 signaling plays a key protective role against BL-induced oxidative stress. 
Previous studies demonstrated that some materials, such as polyphenol, salvianolic acid, 4-acetoxyphenol, 17-beta-estradiol triterpenoid RTA-408, pinosylvin, alphamangostin, and coconut water, protect cells against oxidative stress via Nrf2 signaling. They showed that these materials upregulate Nrf2 protein expression and concluded that these materials protect cells against oxidative stress by increasing Nrf2 protein expression. However, it is unknown whether Nrf2 protein expression is increased by these materials to rescue cells from oxidative stress or whether the expression is secondarily increased in a manner independent of these materials [26-34]. These materials would be expected to stimulate the Nrf2 pathway and increase Nrf2 protein expression to protect cells against oxidative stress.

A limitation of this study is that we examined only an in vitro biological change. A previous study used $\mathrm{Nrf2}^{-/-}$mice subjected to direct light exposure in vivo and revealed tissue change caused by oxidative stress [54]. Studying the effect of BL exposure on living $N r f 2^{-/-}$mice will provide us with more precise information regarding the adverse effects of $\mathrm{BL}$ exposure on the eyes and the importance of the Nrf2 pathway in protecting eyes during BL exposure.

In conclusion, BL exposure induced ROS generation and caused cell death via apoptosis in RPE cells. The Nrf2 pathway plays a protective role against oxidative stress on $\mathrm{BL}$ exposure. Our findings in this study are meaningful for demonstrating the direct relationship between $\mathrm{BL}$ exposure and Nrf2 signaling, as proved by $N r f 2^{-/-}$in RPE cells.

\section{Competing Interests}

The authors declare that they have no competing interests regarding the publication of this paper.

\section{References}

[1] R. Klein and B. E. K. Klein, "The prevalence of age-related eye diseases and visual impairment in aging: current estimates," Investigative Ophthalmology \& Visual Science, vol. 54, no. 14, pp. ORSF5-ORSF13, 2013.

[2] M. van Lookeren Campagne, J. LeCouter, B. L. Yaspan, and W. Ye, "Mechanisms of age-related macular degeneration and therapeutic opportunities," The Journal of Pathology, vol. 232, no. 2, pp. 151-164, 2014.

[3] M. Saito, T. Iida, M. Kano, and K. Itagaki, “Two-year results of combined intravitreal ranibizumab and photodynamic therapy for retinal angiomatous proliferation," Japanese Journal of Ophthalmology, vol. 60, no. 1, pp. 42-50, 2016.

[4] Y. Nomura and Y. Yanagi, "Intravitreal aflibercept for ranibizumab-resistant exudative age-related macular degeneration with choroidal vascular hyperpermeability," Japanese Journal of Ophthalmology, vol. 59, no. 4, pp. 261-265, 2015.

[5] M. Inoue, K. Kadonosono, A. Arakawa, S. Yamane, and T. Ishibashi, "Long-term outcome of intravitreal pegaptanib sodium as maintenance therapy in Japanese patients with neovascular age-related macular degeneration," Japanese Journal of Ophthalmology, vol. 59, no. 3, pp. 173-178, 2015.

[6] L. S. Lim, P. Mitchell, J. M. Seddon, F. G. Holz, and T. Y. Wong, "Age-related macular degeneration," The Lancet, vol. 379, no. 9827, pp. 1728-1738, 2012.
[7] J. Ambati and B. J. Fowler, "Mechanisms of age-related macular degeneration," Neuron, vol. 75, no. 1, pp. 26-39, 2012.

[8] M. Miyake, M. Saito, K. Yamashiro, T. Sekiryu, and N. Yoshimura, "Complement factor H R1210C among Japanese patients with age-related macular degeneration," Japanese Journal of Ophthalmology, vol. 59, no. 5, pp. 273-278, 2015.

[9] F. Ye, H. Kaneko, Y. Nagasaka et al., "Plasma-activated medium suppresses choroidal neovascularization in mice: a new therapeutic concept for age-related macular degeneration," Scientific Reports, vol. 5, article 7705, 2015.

[10] A. Takeda, J. Z. Baffi, M. E. Kleinman et al., "CCR3 is a target for age-related macular degeneration diagnosis and therapy," Nature, vol. 460, no. 7252, pp. 225-230, 2009.

[11] R. Ijima, H. Kaneko, F. Ye et al., "Interleukin-18 induces retinal pigment epithelium degeneration in mice," Investigative Ophthalmology \& Visual Science, vol. 55, no. 10, pp. 6673-6678, 2014.

[12] H. Kaneko, F. Ye, R. Ijima et al., "Histamine H4 receptor as a new therapeutic target for choroidal neovascularization in agerelated macular degeneration," British Journal of Pharmacology, vol. 171, no. 15, pp. 3754-3763, 2014.

[13] F. Ye, H. Kaneko, Y. Hayashi et al., "Malondialdehyde induces autophagy dysfunction and VEGF secretion in the retinal pigment epithelium in age-related macular degeneration," Free Radical Biology \& Medicine, vol. 94, pp. 121-134, 2016.

[14] A. Matsui, H. Kaneko, S. Kachi et al., "Expression of vascular endothelial growth factor by retinal pigment epithelial cells induced by amyloid- $\beta$ is depressed by an endoplasmic reticulum stress inhibitor," Ophthalmic Research, vol. 55, no. 1, pp. 3744, 2015.

[15] A. K. Kiser, E. K. Deschler, and G. Dagnelie, "Visual function and performance with blue-light blocking filters in age-related macular degeneration," Clinical \& Experimental Ophthalmology, vol. 36, no. 6, pp. 514-520, 2008.

[16] A. E. Fletcher, G. C. Bentham, M. Agnew et al., "Sunlight exposure, antioxidants, and age-related macular degeneration," Archives of Ophthalmology, vol. 126, no. 10, pp. 1396-1403, 2008.

[17] T. H. Margrain, M. Boulton, J. Marshall, and D. H. Sliney, "Do blue light filters confer protection against age-related macular degeneration?" Progress in Retinal and Eye Research, vol. 23, no. 5, pp. 523-531, 2004.

[18] H. R. Taylor, B. Munoz, S. West, N. M. Bressler, S. B. Bressler, and F. S. Rosenthal, "Visible light and risk of age-related macular degeneration," Transactions of the American Ophthalmological Society, vol. 88, pp. 163-178, 1990.

[19] M. Kernt, A. Walch, A. S. Neubauer et al., "Filtering blue light reduces light-induced oxidative stress, senescence and accumulation of extracellular matrix proteins in human retinal pigment epithelium cells," Clinical and Experimental Ophthalmology, vol. 40, no. 1, pp. e87-e97, 2012.

[20] A. King, E. Gottlieb, D. G. Brooks, M. P. Murphy, and J. L. Dunaief, "Mitochondria-derived reactive oxygen species mediate blue light-induced death of retinal pigment epithelial cells," Photochemistry and Photobiology, vol. 79, no. 5, pp. 470-475, 2004.

[21] A. R. Wielgus, R. J. Collier, E. Martin et al., "Blue light induced A2E oxidation in rat eyes-experimental animal model of dry AMD," Photochemical and Photobiological Sciences, vol. 9, no. 11, pp. 1505-1512, 2010.

[22] A. Klettner, "Oxidative stress induced cellular signaling in rpe cells," Frontiers in Bioscience, vol. 4, pp. 392-411, 2012. 
[23] R. Howden, "Nrf2 and cardiovascular defense," Oxidative Medicine and Cellular Longevity, vol. 2013, Article ID 104308, 10 pages, 2013.

[24] A. A. Gupte, C. J. Lyon, and W. A. Hsueh, "Nuclear factor (erythroid-derived 2)-like-2 factor (Nrf2), a key regulator of the antioxidant response to protect against atherosclerosis and nonalcoholic steatohepatitis," Current Diabetes Reports, vol. 13, no. 3, pp. 362-371, 2013.

[25] M. M. Sachdeva, M. Cano, and J. T. Handa, "Nrf2 signaling is impaired in the aging RPE given an oxidative insult," Experimental Eye Research, vol. 119, pp. 111-114, 2014.

[26] K. Manna, A. Khan, D. Kr Das et al., "Protective effect of coconut water concentrate and its active component shikimic acid against hydroperoxide mediated oxidative stress through suppression of NF- $\kappa$ B and activation of Nrf2 pathway," Journal of Ethnopharmacology, vol. 155, no. 1, pp. 132-146, 2014.

[27] W. Liu, Z. Xu, T. Yang et al., “The protective role of tea polyphenols against methylmercury-induced neurotoxic effects in rat cerebral cortex via inhibition of oxidative stress," Free Radical Research, vol. 48, no. 8, pp. 849-863, 2014.

[28] H. Zhang, Y.-Y. Liu, Q. Jiang et al., "Salvianolic acid a protects RPE cells against oxidative stress through activation of Nrf2/HO-1 signaling," Free Radical Biology \& Medicine, vol. 69, pp. 219-228, 2014.

[29] J. Hanus, A. Kolkin, J. Chimienti, S. Botsay, and S. Wang, "4Acetoxyphenol prevents RPE oxidative stress-induced necrosis by functioning as an NRF2 stabilizer," Investigative Ophthalmology \& Visual Science, vol. 56, no. 9, pp. 5048-5059, 2015.

[30] C. Zhu, S. Wang, B. Wang et al., "17 $\beta$-Estradiol up-regulates Nrf2 via PI3K/AKT and estrogen receptor signaling pathways to suppress light-induced degeneration in rat retina," Neuroscience, vol. 304, pp. 328-339, 2015.

[31] X. Liu, K. Ward, C. Xavier et al., “The novel triterpenoid RTA 408 protects human retinal pigment epithelial cells against $\mathrm{H}_{2} \mathrm{O}_{2}$-induced cell injury via NF-E2-related factor 2 (Nrf2) activation," Redox Biology, vol. 8, pp. 98-109, 2016.

[32] S. Koinzer, K. Reinecke, T. Herdegen, J. Roider, and A. Klettner, "Oxidative stress induces biphasic ERK1/2 activation in the RPE with distinct effects on cell survival at early and late activation," Current Eye Research, vol. 40, no. 8, pp. 853-857, 2015.

[33] A. Koskela, M. Reinisalo, J. M. T. Hyttinen, K. Kaarniranta, and R. O. Karjalainen, "Pinosylvin-mediated protection against oxidative stress in human retinal pigment epithelial cells," Molecular Vision, vol. 20, pp. 760-769, 2014.

[34] Y. Fang, T. Su, X. Qiu et al., "Protective effect of alpha-mangostin against oxidative stress induced-retinal cell death," Scientific Reports, vol. 6, Article ID 21018, 2016.

[35] Z. Zhao, Y. Chen, J. Wang et al., "Age-related retinopathy in NRF2-deficient mice," PLoS ONE, vol. 6, no. 4, Article ID e19456, 2011.

[36] Z. Xu, H. Cho, M. J. Hartsock et al., "Neuroprotective role of Nrf2 for retinal ganglion cells in ischemia-reperfusion," Journal of Neurochemistry, vol. 133, no. 2, pp. 233-241, 2015.

[37] Z. Xu, Y. Wei, J. Gong et al., "NRF2 plays a protective role in diabetic retinopathy in mice," Diabetologia, vol. 57 , no. 1 , pp. 204-213, 2014.

[38] N. Himori, K. Yamamoto, K. Maruyama et al., "Critical role of Nrf2 in oxidative stress-induced retinal ganglion cell death," Journal of Neurochemistry, vol. 127, no. 5, pp. 669-680, 2013.

[39] T. Kojima, M. Dogru, A. Higuchi et al., "The effect of Nrf2 knockout on ocular surface protection from acute tobacco smoke exposure: evidence from Nrf2 knockout mice," The American Journal of Pathology, vol. 185, no. 3, pp. 776-785, 2015.

[40] H. Cho, M. J. Hartsock, Z. Xu, M. He, and E. J. Duh, "Monomethyl fumarate promotes Nrf2-dependent neuroprotection in retinal ischemia-reperfusion," Journal of Neuroinflammation, vol. 12, no. 1, article 239, 2015.

[41] H. Kaneko, S. Dridi, V. Tarallo et al., "DICER1 deficit induces Alu RNA toxicity in age-related macular degeneration," Nature, vol. 471, no. 7338, pp. 325-332, 2011.

[42] D. Yang, S. G. Elner, L.-R. Lin, V. N. Reddy, H. R. Petty, and V. M. Elner, "Association of superoxide anions with retinal pigment epithelial cell apoptosis induced by mononuclear phagocytes," Investigative Ophthalmology \& Visual Science, vol. 50, no. 10, pp. 4998-5005, 2009.

[43] K. Itoh, T. Chiba, S. Takahashi et al., "An Nrf2/small Maf heterodimer mediates the induction of phase II detoxifying enzyme genes through antioxidant response elements," Biochemical and Biophysical Research Communications, vol. 236, no. 2, pp. 313322, 1997.

[44] F. Ye, T. Zhao, X. Liu et al., "Long-term autophagy and nrf2 signaling in the hippocampi of developing mice after carbon ion exposure," Scientific Reports, vol. 5, article 18636, 2015.

[45] B. Dasari, J. R. Prasanthi, G. Marwarha, B. B. Singh, and O. Ghribi, "The oxysterol 27-hydroxycholesterol increases $\beta$ amyloid and oxidative stress in retinal pigment epithelial cells," BMC Ophthalmology, vol. 10, no. 1, article 22, 2010.

[46] K. Takayama, T. Sato, Y. Karasawa, S. Sato, M. Ito, and M. Takeuchi, "Phototoxicity of indocyanine green and brilliant blue $\mathrm{G}$ under continuous fluorescent illumination on cultured human retinal pigment epithelial cells," Investigative Ophthalmology \& Visual Science, vol. 53, no. 11, pp. 7389-7394, 2012.

[47] R. D. Glickman, "Phototoxicity to the retina: mechanisms of damage," International Journal of Toxicology, vol. 21, no. 6, pp. 473-490, 2002.

[48] S. Proulx, S. Landreville, S. L. Guérin, and C. Salesse, "Integrin $\alpha 5$ expression by the ARPE-19 cell line: comparison with primary RPE cultures and effect of growth medium on the $\alpha 5$ gene promoter strength," Experimental Eye Research, vol. 79, no. 2, pp. 157-165, 2004.

[49] C. S. Alge, S. M. Hauck, S. G. Priglinger, A. Kampik, and M. Ueffing, "Differential protein profiling of primary versus immortalized human RPE cells identifies expression patterns associated with cytoskeletal remodeling and cell survival," Journal of Proteome Research, vol. 5, no. 4, pp. 862-878, 2006.

[50] A. D. Patel, S. Rotenberg, R. L. W. Messer et al., "Blue light activates phase 2 response proteins and slows growth of A431 epidermoid carcinoma xenografts," Anticancer Research, vol. 34, no. 11, pp. 6305-6313, 2014.

[51] C. Huang, P. Zhang, W. Wang et al., "Long-term blue light exposure induces RGC-5 cell death in vitro: involvement of mitochondria-dependent apoptosis, oxidative stress, and MAPK signaling pathways," Apoptosis, vol. 19, no. 6, pp. 922932, 2014.

[52] A. Hirota, Y. Kawachi, K. Itoh et al., "Ultraviolet A irradiation induces NF-E2-related factor 2 activation in dermal fibroblasts: protective role in UVA-induced apoptosis," The Journal of Investigative Dermatology, vol. 124, no. 4, pp. 825-832, 2005.

[53] A. Hirota, Y. Kawachi, M. Yamamoto, T. Koga, K. Hamada, and F. Otsuka, "Acceleration of UVB-induced photoageing in $n r f 2$ gene-deficient mice," Experimental Dermatology, vol. 20, no. 8, pp. 664-668, 2011. 
[54] E. V. Knatko, S. H. Ibbotson, Y. Zhang et al., "Nrf2 activation protects against solar-simulated ultraviolet radiation in mice and humans," Cancer Prevention Research, vol. 8, no. 6, pp. 475486, 2015.

[55] C. L. Saw, M.-T. Huang, Y. Liu, T. O. Khor, A. H. Conney, and A.N. Kong, "Impact of Nrf2 on UVB-induced skin inflammation/ photoprotection and photoprotective effect of sulforaphane," Molecular Carcinogenesis, vol. 50, no. 6, pp. 479-486, 2011. 


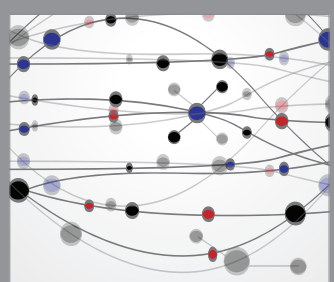

The Scientific World Journal
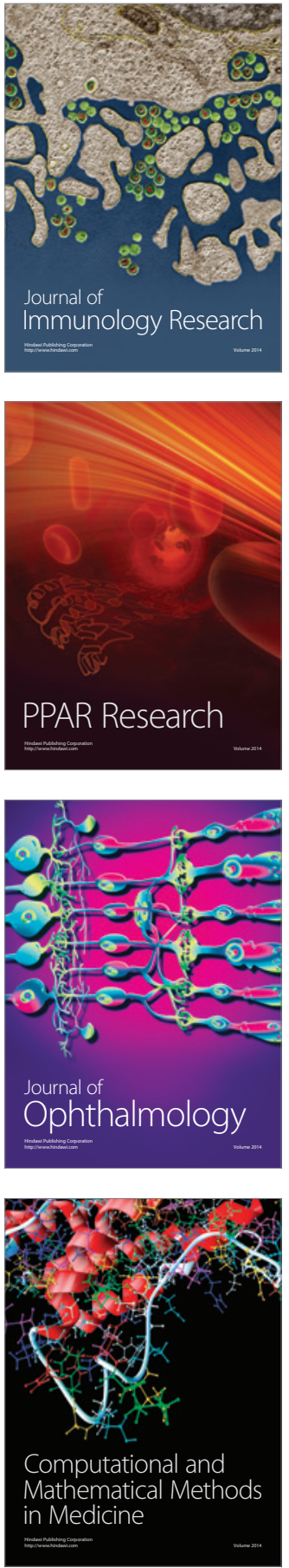

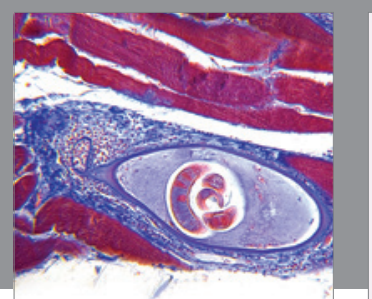

Gastroenterology Research and Practice

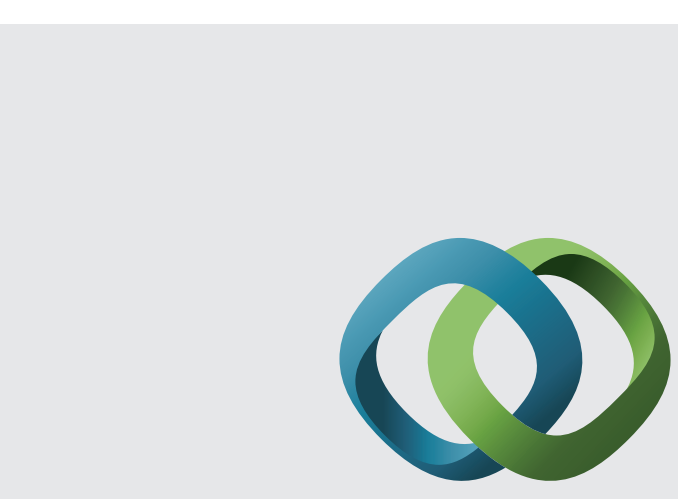

\section{Hindawi}

Submit your manuscripts at

http://www.hindawi.com
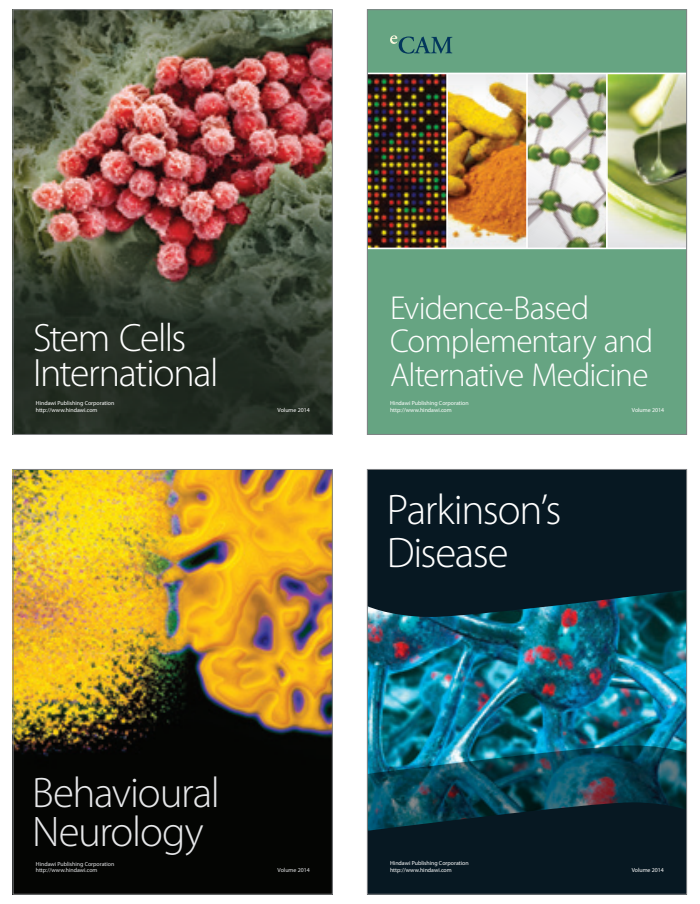
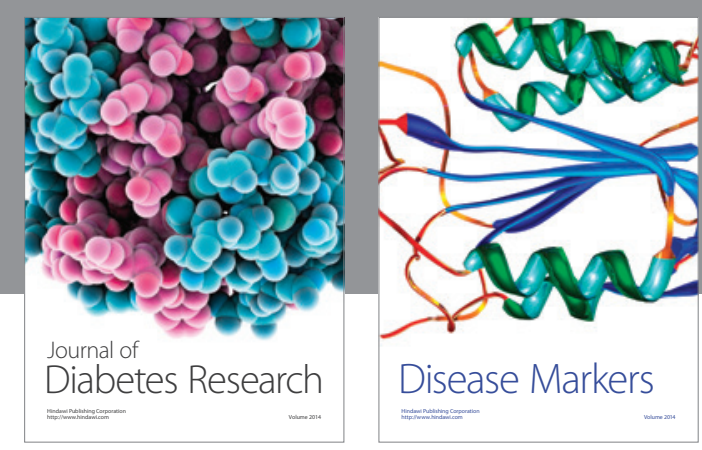

Disease Markers
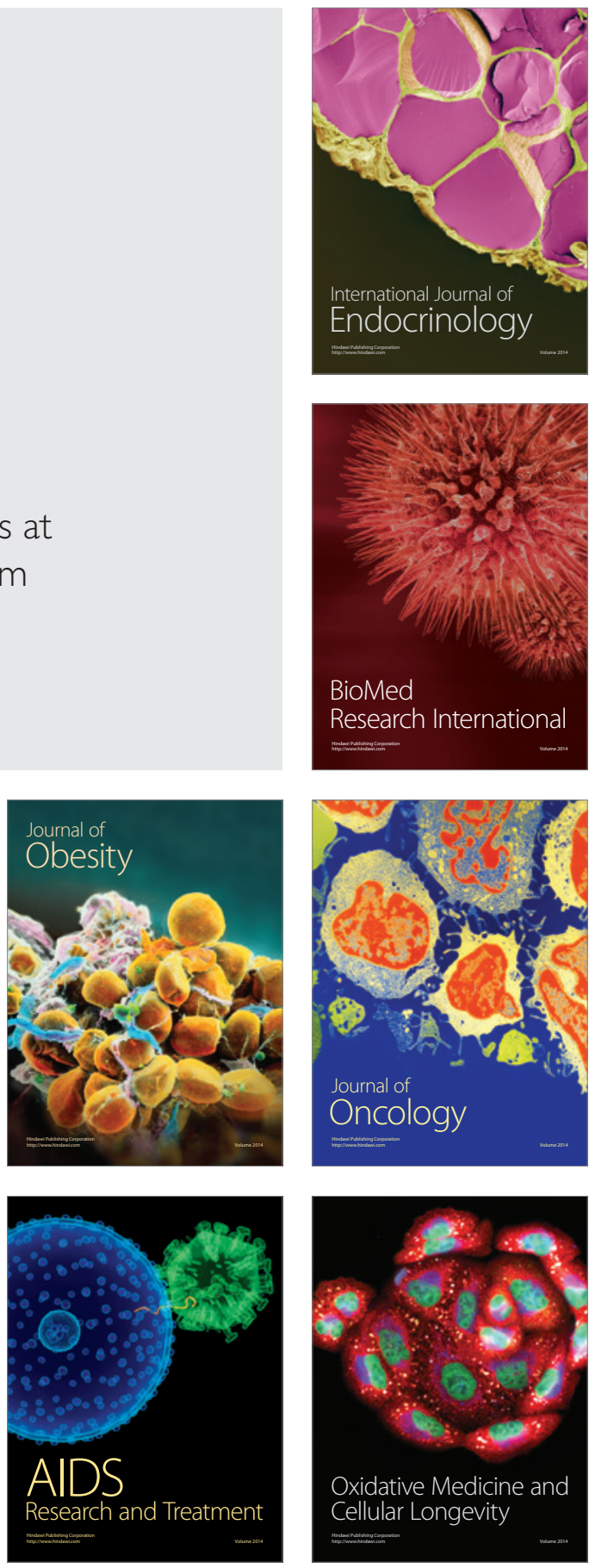